\title{
Mission analysis and preliminary spacecraft design of the enhanced $x$ - ray timing and polarimetry observatory
}

Chen, Wen, Zhu, Zhencai, Xu, Yupeng, Han, Xingbo, Chen, Yehai, et al.

Wen Chen, Zhencai Zhu, Yupeng Xu, Xingbo Han, Yehai Chen, Xingzi Bi, Qi Shi, Rui Liu, Xiangyu Chao, Cheng Zhu, Weiwei Zhao, Shuangnan Zhang, Fangjun Lu, Marco Feroci, Margarita Hernanz, Huili Yuan, Yingquan Yang, Junwang He, Yuan Fang, Yueting Zhang, Xingjian Shi, Kuoxiang Zhang, Jinpei Yu, "Mission analysis and preliminary spacecraft design of the enhanced x-ray timing and polarimetry observatory," Proc. SPIE 11444, Space Telescopes and Instrumentation 2020: Ultraviolet to Gamma Ray, 114442E (13 December 2020); doi: 10.1117/12.2562138 


\title{
Mission analysis and preliminary spacecraft design of the enhanced X- ray Timing and Polarimetry Observatory
}

\author{
CHEN Wen*a, ZHU Zhencai ${ }^{\mathrm{a}}$, XU Yupeng ${ }^{\mathrm{b}}$, HAN Xingbo ${ }^{\mathrm{a}}$, CHEN Yehai ${ }^{\mathrm{a}}$, BI Xingzi ${ }^{\mathrm{a}}$, SHI Qi ${ }^{\mathrm{a}}$, \\ LIU Rui ${ }^{\mathrm{a}}$, CHAO Xiangyu ${ }^{\mathrm{a}}$, ZHU Cheng ${ }^{\mathrm{a}}$, ZHAO Weiwei ${ }^{\mathrm{a}}$, ZHANG Shuangnan ${ }^{\mathrm{b}}$, LU Fangjun ${ }^{\mathrm{b}}$, \\ Marco FEROCI ${ }^{\mathrm{c}}$, Margarita HERNANZ ${ }^{\mathrm{d}}$, YUAN Huili ${ }^{\mathrm{a}}$, YANG Yingquan ${ }^{\mathrm{a}}$, HE Junwang ${ }^{\mathrm{a}}$, FANG \\ Yuan $^{\mathrm{a}}$, ZHANG Yueting ${ }^{\mathrm{a}}$, SHI Xingjian ${ }^{\mathrm{a}}$, ZHANG Kuoxiang ${ }^{\mathrm{a}}$, YU Jinpei ${ }^{\mathrm{a}}$ \\ annovation Academy for Microsatellites of CAS, No. 99 of Haike Road, Shanghai, China; 'Institute \\ of High Energy Physics, CAS, 19B Yuquan Road, Beijing, China; ' Istituto di Astrofisica e \\ Planetologia Spaziale, Istituto Nazionale di Astrofisica, Area di Ricerca di Roma - Tor Vergata, Via \\ Fosso del Cavaliere 100, Roma, I-00133 ITALY; ${ }^{\mathrm{d}}$ Institut de Ciencies de l'Espai, Campus UAB \\ C/ Can Magrans, s/n, 08193 Cerdanyola del Valles (Barcelona), Spain
}

\begin{abstract}
The enhanced X-ray Timing and Polarimetry Observatory (eXTP) is a flagship international collaboration mission led by Chinese Academy of Sciences, with a large contribution from more than 20 European institutes. eXTP mission is designed to study the equation of state of ultra-dense matter under extreme conditions of strong density, gravity and magnetic field. The satellite carries four main instruments, including the Spectroscopy Focusing Array (SFA), the Large Area Detector (LAD), the Polarimetry Focusing array (PFA) and the Wide Field Monitor (WFM), enabling simultaneous spectral-timing-polarimetry studies of celestial sources in the energy range from $0.5-30 \mathrm{keV}$. The satellite will fly at a near-zero-inclination Low Earth Orbit, and is featured with long-time steady high-precision coaxial pointing, near realtime burst alert distribution, and follow-up maneuver capabilities.

This paper describes the primary mission requirements and constraints, and presents an overall mission analysis including orbit analysis, pointing strategy, and board-ground communications, etc. The preliminary design of eXTP satellite is also introduced, including satellite overall configuration, observation modes, avionics architecture and development plan.
\end{abstract}

Keywords: eXTP satellite, X-ray telescope, mission analysis, spacecraft design

\section{INTRODUCTION}

The enhanced X-ray Timing and Polarimetry Observatory (eXTP) is a flagship international collaboration mission led by Chinese Academy of Sciences (CAS), with a large contribution from more than 20 European institutes. eXTP ${ }^{1}$ mission is designed to study the equation of state of ultra-dense matter, to explore the conditions of strong-field gravity, to explore the astrophysics and physics of strong magnetic fields.

eXTP is an enhanced mission concept based on the XTP² mission, enabled by the collaboration between Chinese and European institutions. The predecessor of eXTP, the XTP mission concept, has been selected and funded as one of the so-called background missions in the Strategic Priority Space Science Program of the Chinese Academy of Sciences since 2011. Also in 2011, the Large Observatory for Timing (LOFT ${ }^{3}$ ) mission concept was selected for an assessment study in the context of the ESA's Announcement of Opportunity for the third of the medium size missions (M3) foreseen in the framework of the Agency's Cosmic Vision programme. The LOFT study was carried out in 2011-2014 by a consortium of European institutes. In 2015, the European LOFT consortium and the Chinese team merged the LOFT and XTP mission concepts, thus starting the eXTP project. After merging, the eXTP mission has carried out an extended phase A study in 2018, and officially kicked-off project Phase B in November 2019 as a major background project of CAS.

*wenchen@ustc.edu; phone 8621 62131004; fax 862150735020

Space Telescopes and Instrumentation 2020: Ultraviolet to Gamma Ray, edited by Jan-Willem A. den Herder

Shouleh Nikzad, Kazuhiro Nakazawa, Proc. of SPIE Vol. 11444, 114442E · () 2020 SPIE

CCC code: $0277-786 X / 20 / \$ 21 \cdot$ doi: $10.1117 / 12.2562138$ 
The eXTP international consortium is led by the Institute of High Energy Physics (IHEP) of CAS, and includes other major institutions of CAS, several Chinese Universities, and institutions from ten European countries. Other international partners participate in eXTP as well. Overall more than 200 scientists in over 100 institutions from about 20 countries are members of the consortium. The satellite system is responsible by Innovation Academy for Microsatellites of CAS. The planned launch date of the mission is 2027.

In this paper, we introduce the primary mission requirements and constraints, and present mission analysis and the preliminary design of eXTP satellite.

\section{MISSION OVERVIEW}

\subsection{Scientific objectives}

eXTP is designed to study the equation of state of ultra-dense matter, to explore the conditions of strong-field gravity, to explore the astrophysics and physics of strong magnetic fields. The primary objectives are:

- Determination of the equation of state of matter at supra-nuclear density;

- Measurement of Quantum Electrodynamics (QED) effects in highly magnetized star;

- Investigation of accretion in the strong-field regime of gravity;

- Study of the properties of various X-ray emitting objects.

In addition to investigating fundamental physics, eXTP will be a very powerful observatory for astrophysics that will provide observations of unprecedented quality on a variety of galactic and extragalactic objects. In particular, its wide field monitoring capabilities will be highly instrumental to detect the electro-magnetic counterparts of gravitational wave sources.

\subsection{System architecture}

According to Space Science Programs of CAS, the eXTP mission consists of six systems. They are satellite system, launcher system, launch site system, TT\&C system, ground support system, and science application system.

- The eXTP satellite will incorporate of a Chinese platform supporting the eXTP payloads.

- The eXTP payload consists four types of science instruments from parties, plus equipment and sub-systems.

- Chinese side will provide the Spectroscopy Focusing Array (SFA) and the Polarimetry Focusing Array (PFA). European side will provide the Large Area Detector (LAD) and the Wide Field Monitor (WFM).

- The launcher system, launch site system and TT\&C system are provided by CAS.

- The ground support system is provided by CAS, including X-band ground stations provided by each party. The mission also envisage the use of Beidou Short Message Service for real-time board-to-ground communication, and VHF network for the alert reception provided by European party.

- The science application system is provided by CAS, including science data centers provided by each party.

- The overall eXTP mission will be designed and implemented under CAS's responsibility.

The system architecture is presented in Figure 1. 


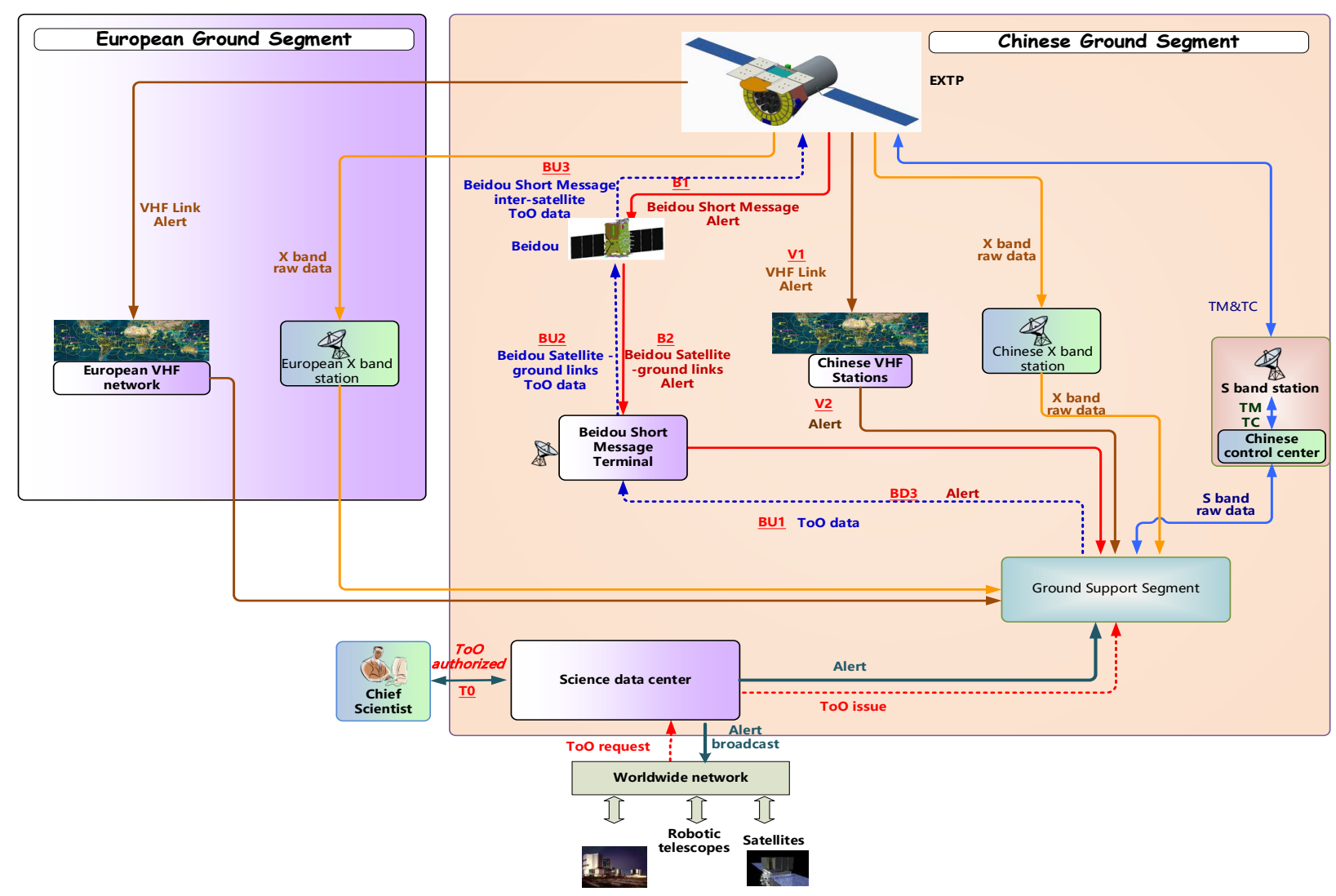

Figure 1. eXTP system architecture

\section{SCIENTIFIC INSTRUMENTS}

The satellite carries four main instruments, including the Spectroscopy Focusing Array (SFA), the Large Area Detector (LAD), the Polarimetry Focusing array (PFA) and the Wide Field Monitor (WFM), enabling simultaneous spectraltiming-polarimetry studies of celestial sources in the energy range from $0.5-30 \mathrm{keV}$.

\subsection{Spectroscopy Focusing Array (SFA)}

The SFA consists of an array of 9 identical Wolter-I (parabola + hyperbola) grazing-incidence X-ray telescopes, and is mainly used for spectral and timing observations in the energy range $0.5-10 \mathrm{keV}$. Each telescope consists of the mirror module, the electron deflector, the filter wheel, and the focal plane camera. The SFA total effective area is expected to be larger than $\sim 6000 \mathrm{~cm}^{2}$ at $2 \mathrm{keV}$, and the field of view (FoV) is 12 arcmin in diameter. The SFA uses silicon drift detectors (SDDs) as focal plane detectors. Each telescope includes a 19-cell SDD array, whose energy resolution is less than $180 \mathrm{eV}$ at $6 \mathrm{keV}$. The SFA parameters and specifications are listed in Table 1.

\subsection{Polarimetry Focusing array (PFA)}

The PFA consists of 4 identical telescopes, which are very similar to the telescopes of SFA, optimized for X-ray imaging polarimetry, sensitive in the energy range of 2-8 keV. In synergy with the SFA, the PFA offers spatial, energy, and/or temporal resolved X-ray polarimetry at high sensitivity based on the gas pixel detector (GPD). PFA is also the only instrument on eXTP that has an imaging capability better than an arcminute, which may help to discriminate and remove source confusions occurring in other instruments. The PFA parameters and specifications are listed in Table 2. 
Table 1. SFA main specifications

\begin{tabular}{|l|l|}
\hline \multicolumn{1}{|c|}{ Item } & \multicolumn{1}{c|}{ Requirements } \\
\hline Effective area (given energy is $\mathrm{E} \pm 0.5 \mathrm{keV})$ & $\geqslant 6000 \mathrm{~cm} 2 @(1-2) \mathrm{keV}$ \\
& $\geqslant 4400 \mathrm{~cm} 2 @ 6 \mathrm{keV}$ \\
\hline Energy range & $0.5-10 \mathrm{keV}$ \\
\hline Energy resolution @6keV & $\leqslant 180 \mathrm{eV}$ \\
\hline Field of View (FWHM) & $\varphi \geqslant 12 \mathrm{arcmin}$ \\
\hline Response stability $(\Delta \mathrm{P} / \mathrm{P})$ & $\leq 5 \%$ \\
\hline Point source localization & $\leq 5 \mathrm{arcsec}(1 \sigma)$ \\
\hline Time resolution & $\leq 10 \mu \mathrm{s}$ \\
\hline Absolute time accuracy & $\leq 2 \mu \mathrm{s}$ \\
\hline Dead time & $\leqslant 5 \% @ 1 \mathrm{Crab}$ \\
\hline Background rate & bkg(0.5-10 keV) $\leqslant 0.2 \mathrm{cts} / \mathrm{s}$ \\
\hline Sustained flux & bkg(2-10 keV) $\leqslant 0.1 \mathrm{cts} / \mathrm{s}$ \\
\hline
\end{tabular}

Table 2. PFA main specifications

\begin{tabular}{|l|l|}
\hline \multicolumn{1}{|c|}{ Item } & \multicolumn{1}{c|}{ Requirements } \\
\hline Effective area (given energy is $\mathrm{E} \pm 0.5 \mathrm{keV})$ & $\geq 380 \mathrm{~cm} 2 @ 3 \mathrm{keV}$ \\
\hline Energy range & $2-8 \mathrm{keV}$ \\
\hline Energy resolution @5.9keV $(\Delta \mathrm{E} / \mathrm{E})$ & $\leq 25 \%$ \\
\hline Field of View (FWHM)@3 keV & $\geq 8 \mathrm{x} 8 \mathrm{arcmin}$ \\
\hline Angular Resolution @2 keV & $\leq 30 \operatorname{arcsec}(\mathrm{HPD})$ \\
\hline Response stability $(\Delta \mathrm{P} / \mathrm{P})$ & $\leq 5 \%$ \\
\hline Point source localization & $\leq 5 \mathrm{arcsec}(1 \mathrm{\sigma})$ \\
\hline Time resolution & $\leq 10 \mu \mathrm{s}$ \\
\hline Absolute time accuracy & $\leq 4 \mu \mathrm{s}$ \\
\hline Dead time & $\leqslant 30 \%$ @ Crab \\
\hline Dead time knowledge & $\leq 10 \%$ \\
\hline Background rate & bkg(2-8 keV) $\leq 0.1 \mathrm{cts} / \mathrm{s} / \mathrm{cm} 2 / \mathrm{det}-1$ \\
\hline Maximum flux (sustained) & $\geq 1 \mathrm{Crab}$ \\
\hline Spurious polarisation & $\leq 1 \%$ \\
\hline
\end{tabular}




\subsection{Large Area Detector (LAD)}

$\mathrm{LAD}^{4}$ is a few m2-class instrument with $\sim 10$ times the collecting area of the best past timing missions (such as RXTE, the largest predecessor), which holds the capability to revolutionize the studies of X-ray variability from X-ray sources on the millisecond time scales. The LAD will operate in the energy range 2-30 keV (up to $80 \mathrm{keV}$ in expanded mode) with good spectral resolution ( $<260 \mathrm{eV} @ 6 \mathrm{keV}$ Full Width Half Maximum, FWHM) and a temporal resolution of 10 $\mu$ s. The core performance requirements and goals of LAD are described in Table 3.

Table 3. LAD main specifications

\begin{tabular}{|l|l|}
\hline \multicolumn{1}{|c|}{ Item } & \multicolumn{1}{|c|}{ Requirements } \\
\hline Effective area (given energy is $\mathrm{E} \pm 0.5$ & $\geq 3.0 \mathrm{~m}^{2} @ 8 \mathrm{keV}$ \\
$\mathrm{keV})$ & $\geq 1.5 \mathrm{~m}^{2} @ 2.5 \mathrm{keV}$ \\
& $\geq 0.37 \mathrm{~m}^{2} @ 30 \mathrm{keV}$ \\
\hline Energy range & $2-30 \mathrm{keV}$ primary \\
& $2-80 \mathrm{keV}$ extended \\
\hline Energy Resolution@6keV & $\leq 260 \mathrm{eV}$ (all events) \\
& $\leq 200 \mathrm{eV}$ (singles, end of life) \\
\hline Response Stability & $0.0001-0.01 \mathrm{~Hz}:<3.4 \%$ per decade \\
& $0.01-1 \mathrm{~Hz}:<0.34 \%$ per decade \\
& $1-1200 \mathrm{~Hz}:<0.034 \%$ per octave \\
& $>2000 \mathrm{~Hz}:$ Lower is better \\
& $10-2000 \mathrm{~Hz}:<0.00034 \%$ nearly periodic \\
\hline Time resolution & $10 \mu \mathrm{s}$ \\
\hline Dead time & $\leq 1 \%$ @ $1 \mathrm{Crab}$ \\
\hline Background & $\leq 10 \mathrm{mCrab}$ \\
\hline Max source flux & $\geq 15 \mathrm{Crab}$ \\
\hline
\end{tabular}

The LAD payload is a large array of X-ray detectors, 640 SDDs, electrically and mechanically organized in group of 16 , referred to as Modules, for a total of 40 Modules. Ten Modules are electrically connected to a Panel Back End Electronic (PBEE) and each of them is connected to the Instrument Control Unit (ICU), nominal and redundant. ICU is then connected to the spacecraft. The primary features of LAD are: 1) a large effective area for a single on-axis source; 2) no imaging, but instead collimation to maximize on-axis effective area while rejecting off-axis flux (other sources, background); 3) high energy resolution. These are made possible by developments in detectors and micro-collimator structures.

\subsection{The Wide Field Monitor (WFM)}

WFM is an instrument with the capability to simultaneously observe about $1 / 3$ of the sky $(1.33 * \mathrm{pi}=33 \%$ at $20 \%$ of peak response, and $1.75 * \mathrm{pi}=44 \%$ at zero response) in the same energy band as the LAD. The main purpose of the WFM is to detect sources for follow-up observations with the LAD, SFA and PFA, so the field of view is designed to have a maximum overlap with the sky accessible to LAD, SFA and PFA pointings. These sources are new transients, as well as known sources undergoing spectral state changes.

The WFM is based on a modular design with six identical Cameras. The WFM detector employs the same basic detector technology as the LAD, but with a read-out scheme that enables 2D position determination of the X-ray interactions required for the imaging. However, the detector position resolution in one direction is much finer $(<100 \mu \mathrm{m})$ than in the 
other direction $(<8 \mathrm{~mm})$. This is reflected in the elongated point spread function of the de-convolved sky images. Therefore, the six WFM Cameras are grouped into three Camera Pairs with each two coaligned cameras rotated $90^{\circ}$ around the viewing direction with respect to each other. This combination can achieve the required 1 arcmin source localization accuracy in two dimensions. The three pairs are oriented to cover the required large fraction of the sky. The core performance requirements and goals of WFM are described in Table 4.

Table 4. WFM main specifications

\begin{tabular}{|c|c|}
\hline Item & Requirements \\
\hline $\begin{array}{l}\text { Point source localization } \\
\text { (confidence level 90\%) }\end{array}$ & $\leq 1 \operatorname{arcmin}$ \\
\hline Angular resolution & $\leq 5$ arcmin \\
\hline $\begin{array}{l}\text { Peak sensitivity } \\
\text { detection) }\end{array}$ & $\begin{array}{l}1 \text { Crab (1 s) } \\
5 \text { mCrab (50 ks) }\end{array}$ \\
\hline Field of view & $\geq 3.1 \mathrm{sr}$ (around all the pointing instruments) \\
\hline Energy range & $2-50 \mathrm{keV}$ \\
\hline Energy resolution & $\leq 500 \mathrm{eV}$ \\
\hline Absolute time calibration accuracy & $2 \mu s$ \\
\hline Number of triggers for WFM & $\geq 5$ per day \\
\hline On-board memory & 5 min @100 Crab \\
\hline
\end{tabular}

\section{MISSION ANALYSIS}

\subsection{Orbit selection}

The Orbit selection of eXTP mission is derived from LAD energy resolution requirement (260eV@6keV), which is related to instrument background rates, stability and radiation damage due to charged particles. Space irradiation simulation and detector particle irradiation test for LAD instrument has been performed during the pre-research phase of the LOFT project and results was shown in Figure 2. According to the figure, the orbital proton fluence during the lifetime must be less than $4 \times 10^{-7} \mathrm{p} / \mathrm{cm}^{2}$ to meet $\mathrm{LAD}$ energy resolution requirement.

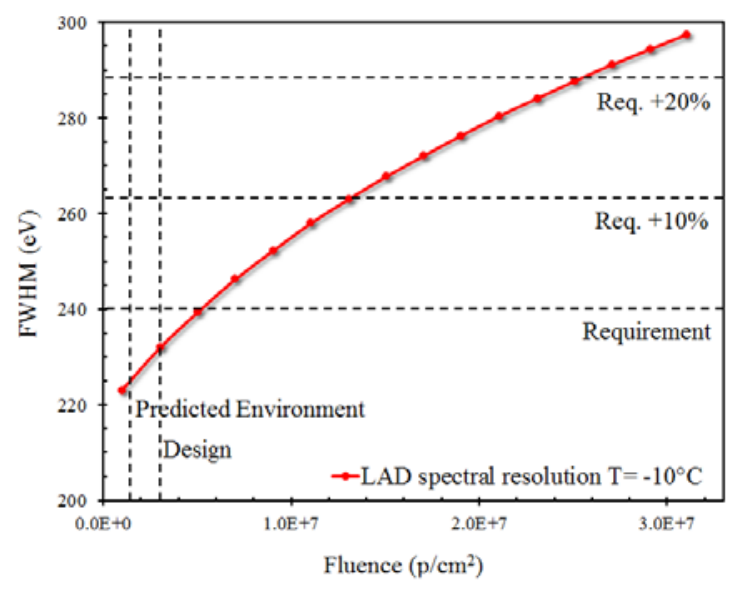

Figure 2. The relationship between energy resolution and radiation dose

Only a LEO orbit provides a low enough background. In combination with a low inclination the radiation damage to the detectors will be low enough to have them passively cooled and still reach the end-of-life temperature requirements. 
Combining analysis of the orbital proton fluence through the AE9 and AP9 models, the orbital height should be less than $550 \mathrm{~km}$ and the orbital inclination angle should be less than $2.5^{\circ}$. In addition, the background of the detector is interfered by the background of particles, and the background of the particles is affected by geomagnetic stiffness and SAA. The circular orbit is beneficial to the stability of background and suppresses the background system error for all instruments.

Therefore, the orbit of eXTP is chosen as:

- Orbit height: $\leqslant 550 \mathrm{~km}$;

- Orbital inclination: $\leqslant 2.5^{\circ}$;

- Eccentricity: 0;

\subsection{Observation program}

eXTP observation program contains the Routine Program Observation, Burst Pointing Observation, Targets of Opportunity (ToO) Observation, and Calibration Observation.

1. Routine Program is dedicated to observations of already known sources, selected by the scientists, pre-planned and uploaded to the spacecraft

2. Burst Observation is dedicated to the burst sources detected and localised onboard by the wide field of view WFM. By pre-defined priority comparison algorithm, the spacecraft autonomously slews upon the burst detection and points to burst sources for timely observation.

3. Targets of Opportunity (ToO) are observations of scientific events that cannot be foreseen and whose scientific importance warrants immediate alteration of the current routine program. ToO request is proposed by the scientists and timely uploaded to the spacecraft for observation.

4. Instrument Calibrations: a full scientific characterization of the instruments is to be performed at regular intervals (frequency depending on instruments and status) through the course of the mission. Spacecraft is scheduled to point to known sources for instrument performance calibration.

These four observation programs and the transition strategy onboard are depicted in the Figure 3.

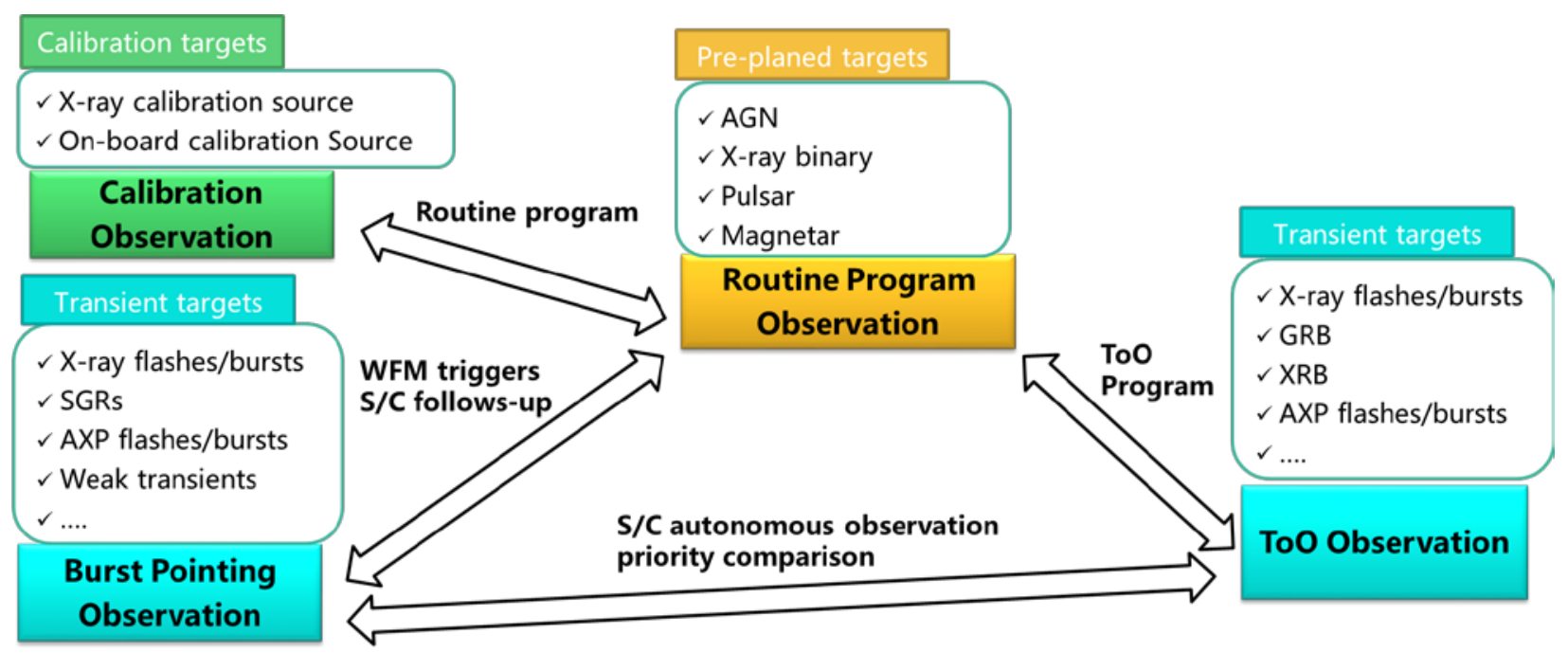

Figure 3. eXTP observation programs

\subsection{Pointing strategy}

Pointing strategy of eXTP is driven by the constraints of payload observable sky area, avoidance angle of Sun/Earth/Moon with regard to instrument boresight, and thermal design optimisation. Define the nominal optical axis of 
SFA、 PFA and LAD as $+X$ axis, shown in Figure 4. To optimize the thermal flux environment of the satellite, the Sun vector is constrained in the $\mathrm{XOZ}$ plane with $+\mathrm{Z}$ direction. The mission requires $50 \%$ of the sky is observable. After visible sky coverage analysis, the angle between the sun and $+X$ axis should be greater than $60^{\circ}$, the angle between the sun and $+\mathrm{X}$ axis should less than $<120^{\circ}$. As

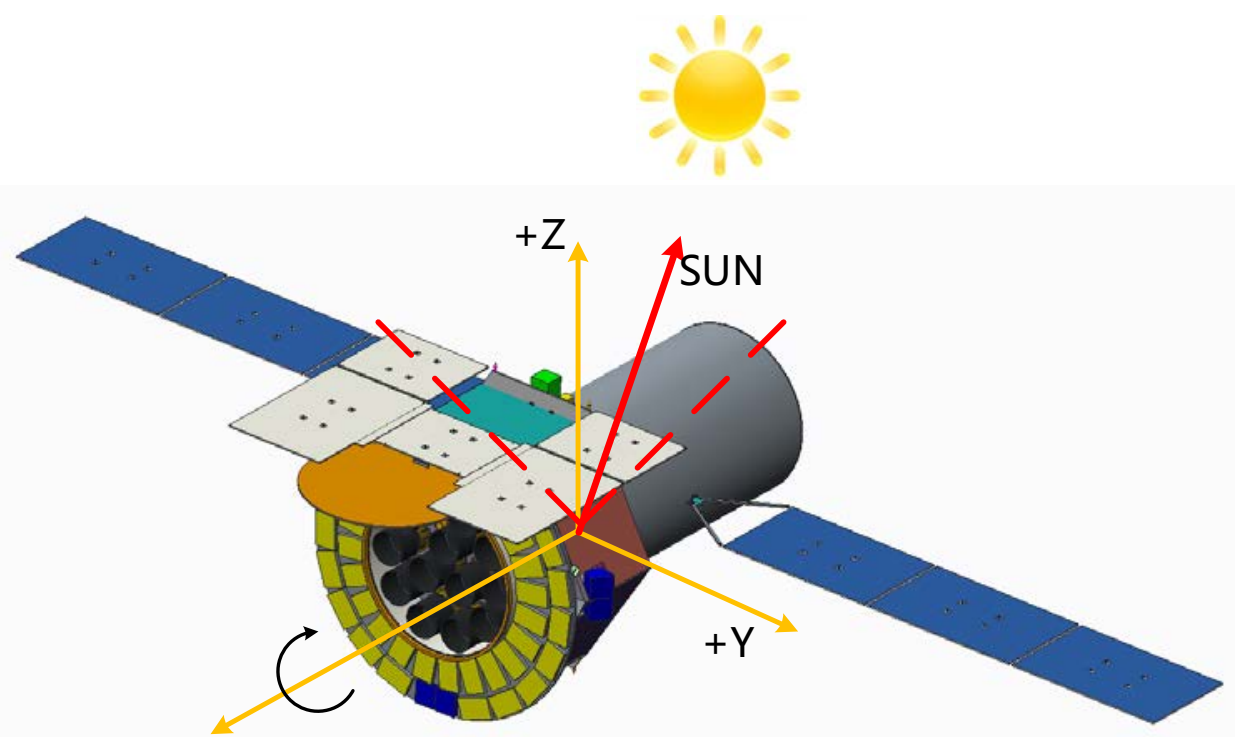

Figure 4. eXTP satellite configuration and pointing strategy

Therefore the eXTP pointing strategy follows rules as below:

1. the angle between the sun and $60^{\circ}<+X<120^{\circ}$;

2. S/C maneuvers around the $\mathrm{X}$ axis and the sun vector is constrained in the $\mathrm{XOZ}$ plane $+\mathrm{Z}$ direction. The angle between the sun and $-\mathrm{Z}>90^{\circ}$ and the angle between the sun and the XOZ plane is less than $\pm 2^{\circ}$.

With the above rules, there are many advantages for satellite. First, thermal flux at the surface $( \pm Y,-Z$ axis) of satellite is very stable, ideal for heat radiation. At the same time, it also good for solar array with adjustment mechanism to hold the sun vertical to the solar array, to achieve best power supply in any pointing direction. With such pointing strategy, it's easy to implement sun shade for instruments. It's also convenient for satellite to maneuver from one observation pointing to another, maximum 60 degrees around space axis.

\subsection{Board-to-ground communication}

As shown in Figure 1, there are 3 types of board-to-ground communication for eXTP satellite. S-band is adopted for satellite telecommand and telemetry communication. X-band is used for science data downlink, where both Chinese and European X-band ground stations are to be used for receiving science telemetry. Besides the huge data volume requirements, there are also real-time communication requirements. Fast ToO observation plan upload is expected to be realized through Chinese Beidou Short Message Service. For real-time downlink, it is required to quickly pass the burst information to the ground. From the moment when the S/C receives the trigger Alert information (including the trigger time and the location of the transient source) generated by the WFM, the data processing should be completed within 30s and then downlinked to the ground support system. Two approaches are currently considered for the real-time downlink:

1. eXTP satellite $\rightarrow$ VHF ground station $\rightarrow$ Ground support system $\rightarrow$ Scientific application system;

2. eXTP satellite $\rightarrow$ Beidou satellite $\rightarrow$ Beidou Ground Operation Control Center $\rightarrow$ Scientific Application System. 


\section{SATELLITE PRELIMINARY DESIGN}

\subsection{Satellite Configuration}

The configuration of eXTP satellite is shown in Figure 4. The satellite consists of three modules, which are optical module, service module and detector module. The SFA/PFA mirror assemblies, LAD modules and one pair of WFM cameras are accommodated in optical module on the $+X$ side of the satellite, with the instruments boresight pointing to the $+\mathrm{X}$ direction. Service module contains platform avionics and AOCS equipment, the metering structure tube, as well as two pairs of WFM cameras. The WFM cameras are placed to guarantee that there is no obstacle in their wide field of view, as shown in Figure 5. The detector electronics of SFA and PFA are accommodated in the detector module. The sunshield is on the $+\mathrm{Z}$ side to protect the payloads from sunlight. The solar arrays are on the \pm Ys side of the satellite. The overall size of the eXTP satellite (in-orbit status) is about $9365 \mathrm{~mm}(\mathrm{X}) * 20092 \mathrm{~mm}(\mathrm{Y}) * 4383 \mathrm{~mm}(\mathrm{Z})$. The total mass is about $5200 \mathrm{~kg}$.

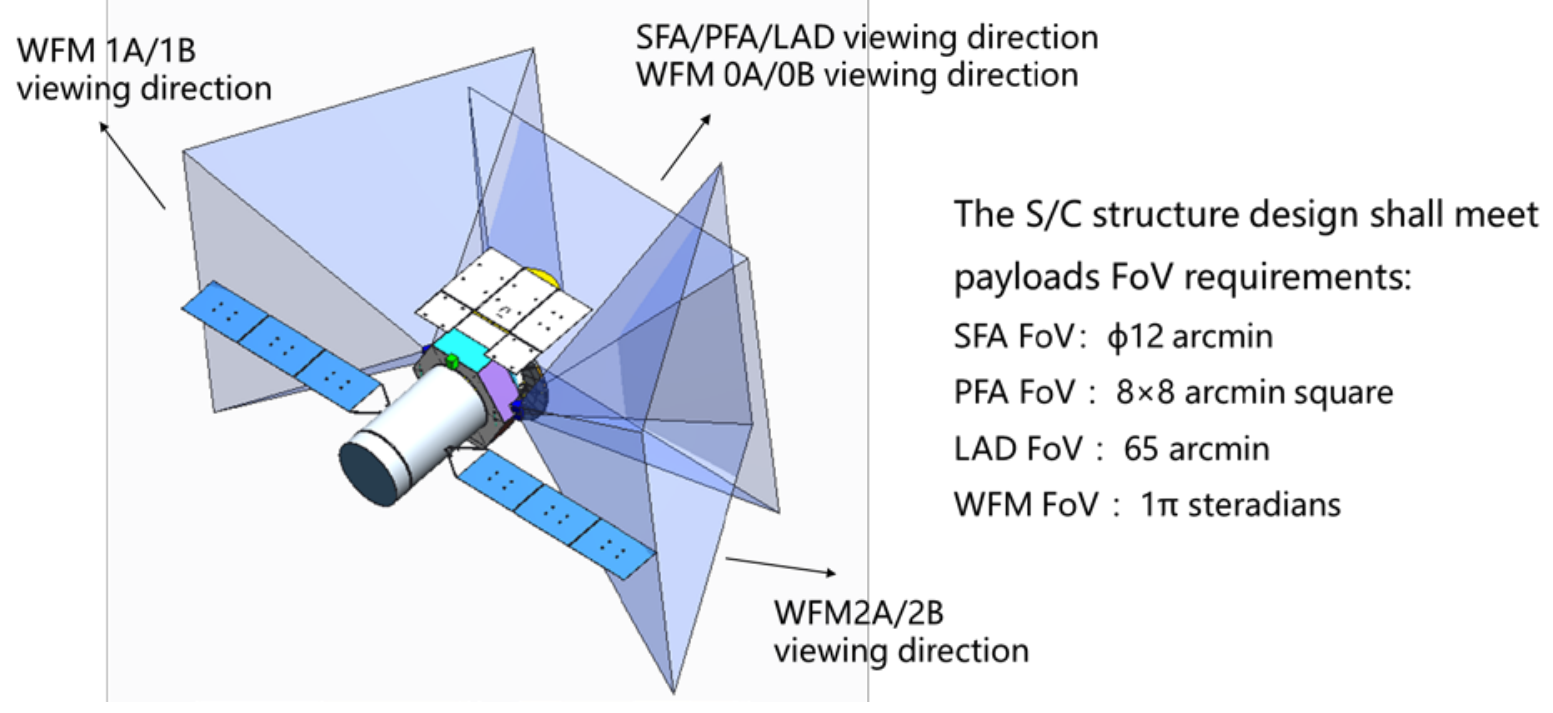

Figure 5. Field of view of eXTP instruments

\subsection{Thermal Control Design}

The task of thermal control subsystem is to keep the temperature of all the instruments on board in the required range. The eXTP satellite mainly adopt passive thermal dissipation and active heating control strategy, using radiators with high efficiency to dissipate the heat generated by equipment and instruments to the outer space. Pre-embedded heat pipes are used to enhance the heat exchange process inside the modules. For the optical module, the main task is to maintain the SFA/PFA mirror assemblies a very stable temperature around $20 \pm 1^{\circ} \mathrm{C}$, to decrease thermal deformation caused by temperature inequality. At the same time, large thermal dissipation bottom surface of LAD modules needs to be kept obstacle-free to assist the thermal exchange with out space. The optical bench and its supporting structure of the optical module, the main metering structure tube of the service module, and the detector bench of the detector module are all thermally controlled within $20 \pm 2^{\circ} \mathrm{C}$, for maintaining good alignment for instruments. The thermal control requirements for different modules are shown in Figure 6. 


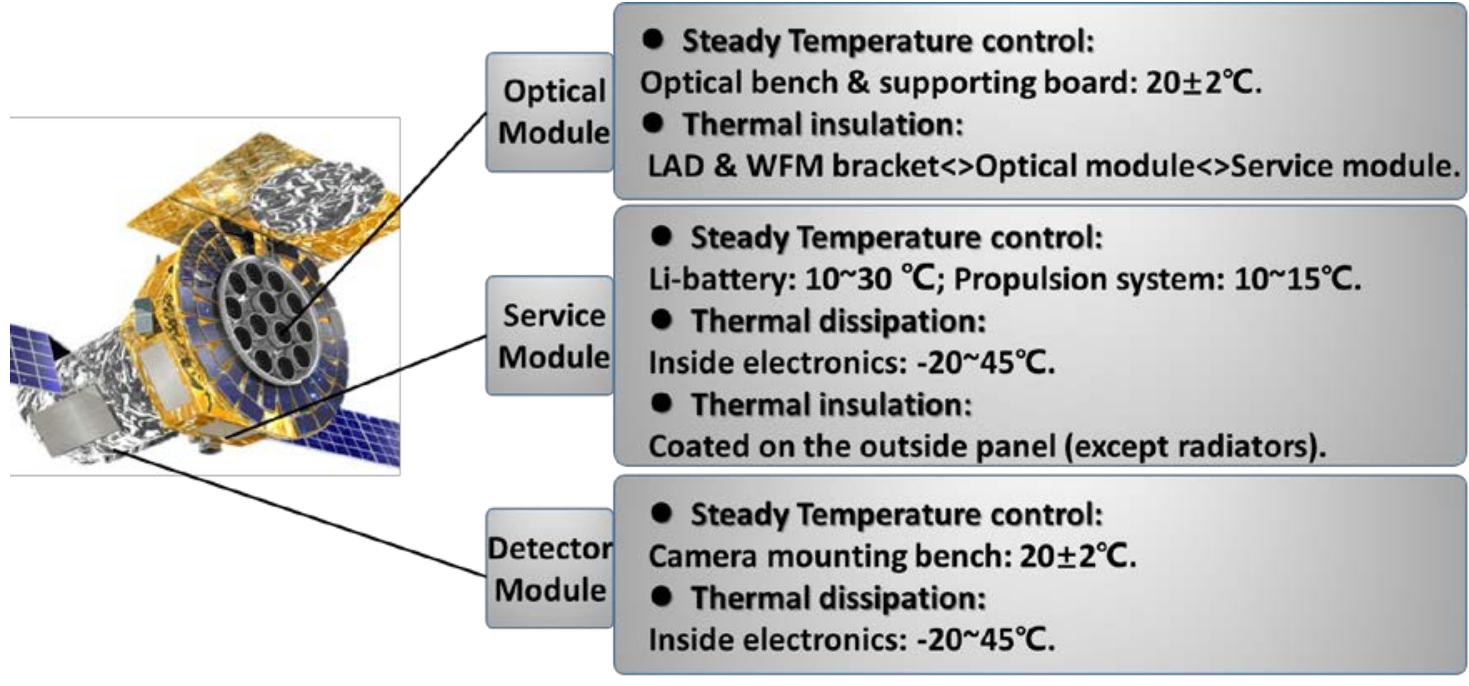

Figure 6. Thermal control requirements of eXTP satellite

\subsection{Avionics Architecture}

The overall avionic architecture of eXTP satellite is illustrated in Figure 7. The power subsystem manages the generation, storage and distribution of the electrical power needed by the spacecraft. The eXTP satellite is equipped with solar panel around $36 \mathrm{~m}^{2}$ and high performance Li-battery is selected for power storage, with overall capacity around 270 Ah. Two power bus lines are adopted, with nominal bus voltage $43 \mathrm{~V}$ and $30 \mathrm{~V}$, respectively.

The eXTP satellite has a centralized data management system, with Satellite Management Unit (SMU) and Payload Data Processing Unit (PDPU) as two core centers. SMU controls and commands activities of most platform units, and fulfil functions including flight dynamics control, AOCS data processing, telemetry control, power and thermal control and so on. PDPU is responsible for payloads management and on-board data management. It communicates with Chinese payload ICUs through the CAN bus and communicates with European payload ICUs through the SpaceWire bus.

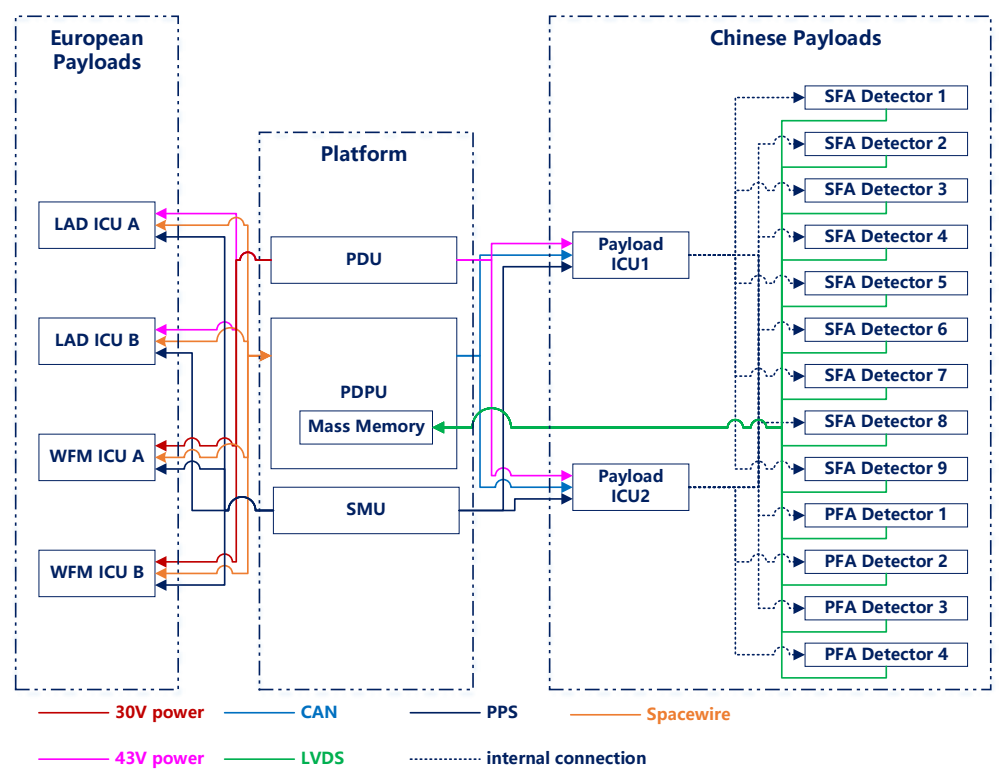

Figure 7. Satellite avionics concept 
PDPU also integrates function of mass memory. Accroding to eXTP observing strategy, onboard storage and communication capability should satisfy the extremely case: 15 Crab BHC outbursts observation for 8 hours every 3 days. In that case, the maximum volume of science data is approximately 3.4Tb for 3 days. In order to handle it, PDPU is designed to store the observation data transmitted through LVDS/spacewire interface by the payload, and communication subsystem transmits data to the ground stations through X-band link. The capacity of onboard storage is $4.5 \mathrm{~Tb}$.

\subsection{Attitude and Orbit Control Subsystem}

The eXTP satellite is a three-axis stabilized spacecraft with high pointing accuracy and stability. The architecture of the attitude and orbit control subsystem includes:

- Actuators:

$\checkmark 8$ reaction wheels;

$\checkmark 6$ torque-rods;

$\checkmark 12$ thrusters;

- Sensors:

$\checkmark 3$ sun sensors;

$\checkmark 2$ three-axial magnetometers;

$\checkmark 2$ star trackers;

$\checkmark 2$ fine guidance sensors;

$\checkmark 2$ gyros;

The attitude control subsystem exploits these sensors and actuators and is capable of attitude determination and stabilised mission pointing specified as follows:

- Attitude Pointing accuracy: $\leqslant 0.01^{\circ}$;

- Attitude Measurement accuracy: $\leqslant 0.002^{\circ}$;

- Pointing stability: $\leqslant 0.005 \%$ s.

\section{DEVELOPMENT PLAN}

Phase B of eXTP project was officially kicked-off in November 2019. With the development duration of 91 months, the satellite is expected to be launched in 2027. Taking Phase B kick-off as T0, the main satellite development planning is as follows:

- Phase B: T0+16m (till 2021.03), until Satellite Preliminary Design Review;

- Phase C1: T0+37m (till 2022.12), mainly for satellite mechanical and thermal model design, development and verification;

- Phase C2: T0+58m (till 2024.09), mainly for satellite qualification model design, development and verification;

- Phase D: T0+88m (till 2027.03), dedicated for satellite flight model development and verification;

- Launch: T0+91m (till 2027.06). 


\section{CONCLUSIONS}

In this paper we have presented the mission analysis and preliminary design of the eXTP satellite. The eXTP mission is designed to address key questions of physics in the extreme conditions of ultra-dense matter, strong field gravity, and the strongest magnetic field existing in nature. The payload includes three narrow field-of-view instruments, SFA, PFA and $\mathrm{LAD}$, as well as wide field-of-view WFM. The satellite flies on an orbit of nearly zero inclination circular orbit with altitude of $550 \mathrm{~km}$. The satellite mainly observes pre-planned targets, and is capable of autonomous slew to burst sources detected onboard, or ToO objects quickly uploaded from the ground, which is made possible through satellite's real-time communication link with the ground.

The mission is led by China, currently supported by Chinese Academy of Sciences and executed under the management of the National Space Science Center of CAS. The project has officially kicked off Phase B in November 2019. After Phase C1, official mission adoption is expected. With Phase C2 and Phase D planned in 2023-2027, the eXTP satellite is targeted to be launched in 2027.

\section{REFERENCES}

[1] ShuangNan Zhang, Andrea Santangelo, Marco Feroci, YuPeng Xu, et al., "The enhanced X-ray Timing and Polarimetry mission---eXTP”, SCIENCE CHINA Physics, Mechanics \& Astronomy, Volume 62 , Issue 2 : 029502(2019).

[2] Y. Dong, et al., "In The X-ray timing and polarization satellite-1, 2, 3: Uncovering the mysteries of black holes and extreme physics in the universe”, Proceedings Volume 9144, Space Telescopes and Instrumentation 2014: Ultraviolet to Gamma Ray.

[3] M. Feroci, J. W. den Herder, E. Bozzo, D. Barret, S. Brandt, M. Hernanz, M. van der Klis, M. Pohl, A. Santangelo, et. Al., "in The large observatory for X-ray timing”, Proceeding SPIE 9144, Space Telescopes and Instrumentation 2014: Ultraviolet to Gamma Ray.

[4] M. Feroci, J. W. den Herder, E. Bozzo, D. Barret,S. Brandt, et al., “The Large Observatory for Timing”, Proceedings of the SPIE, Vol. 9144, 91442T-1, 2014. 